Proceedings

\title{
Highly Stable PEDOT:PSS Coating on Gold Microelectrodes with Improved Charge Injection Capacity for Chronic Neural Stimulation ${ }^{+}$
}

\author{
Anmona S. Pranti *, Andreas Schander, André Bödecker and Walter Lang \\ Institute for Microsensors, -Actuators and -Systems (IMSAS), University of Bremen, 28359 Bremen, Germany; \\ aschander@imsas.uni-bremen.de (A.S.); aboedecker@imsas.uni-bremen.de (A.B.); \\ wlang@imsas.uni-bremen.de (W.L.) \\ * Correspondence: apranti@imsas.uni-bremen.de; Tel.: +49-421-218-62613 \\ + Presented at the Eurosensors 2017 Conference, Paris, France, 3-6 September 2017.
}

Published: 25 August 2017

\begin{abstract}
This study introduces two new processes that highly enable PEDOT:PSS (poly(3,4-ethylenedioxythiophene)-poly(styrenesulfonate)) as stable coating material for chronic neural stimulation. In first process, strong mechanical bonding between PEDOT:PSS coating and gold electrodes is achieved by creating rough porous surface with partial iodine etching. PEDOT:PSS coating on iodine etched gold electrode shows 100\% stability under strong ultrasonic treatment. The second process represents electrochemical modification of PEDOT:PSS coating by cyclic voltammetry method in Ringer's solution. This process reduces electrode polarization 33\% during stimulation. Therefore, charge injection capacity increases that ensures safe stimulation. A combination of both processes facilitates the use of PEDOT:PSS coating for successful chronic neural recording and stimulation.
\end{abstract}

Keywords: PEDOT:PSS; iodine etching; chronic neural stimulation; neural interfaces; long-term stability

\section{Introduction}

Miniaturization and high charge injection capacity of neural interfaces electrodes are the paramount preconditions for successful stimulation and recording signal from specific target cell [1]. The problem associated with small electrode is low charge injection capacity as it is proportional to the surface area of electrode-brain tissue interface [2]. PEDOT:PSS coating is used to increase the surface area of microelectrodes as it provides a rough surface. Consequently, electrode charge injection capacity increases and interface impedance decreases [2,3]. However, poor stability and adhesion to the electrode surface limits its use for long-term neural stimulation, because delamination of electrode material is not preferable for sophisticated physiological application [2]. Therefore improvement of PEDOT:PSS coating adhesion to the electrode is an essential task to use it for chronic bidirectional application (signal recording and stimulation). Conversely, further improvement of charge injection capacity of PEDOT:PSS coated electrode facilitates safe stimulation. Hence electrochemical modification without degrading electronic property of PEDOT:PSS coating increases the possibility of successful neural stimulation.

\section{Materials and Methods}

\subsection{Fabrication of Flexible Electrode Arrays}

Flexible gold electrodes ( $585 \mu \mathrm{m}$ diameter) arrays used for neural interfaces were fabricated for this analysis. Figure 1 shows the general fabrication steps. $500 \mathrm{~nm}$ thick gold conductive paths were 
insulated by $5 \mu \mathrm{m}$ thick polyimide layers on both sides. Additionally $2.5 \mu \mathrm{m}$ thick gold layer was electrodeposited on the connector pads and electrodes to improve mechanical stability. Finally, the flexible electrode arrays were detached from silicon substrate by deep reactive ion etching. The entire fabrication process with more details is reported elsewhere [4].
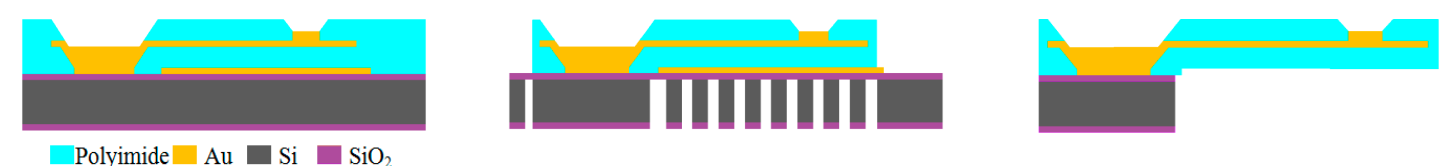

Figure 1. General fabrication steps of flexible gold electrode array: deposition and structuring of gold and polyimide layer by layer (left); deep reactive ion etching of silicon (middle) and releasing of the array by chemical etching of gold sacrificial layer (right).

\subsection{Gold Etching in Iodine Solution}

Gold electrodes were partially etched using iodine solution $0.5 \mathrm{~mol} / \mathrm{L}$ (Grüssing $\mathrm{GmbH}$, Germany) to create a rough porous surface. Etching was performed in different concentration solutions $(0.5 \mathrm{~mol} / \mathrm{L}, 0.1 \mathrm{~mol} / \mathrm{L}, 0.05 \mathrm{~mol} / \mathrm{L})$ for different time duration $(10 \mathrm{~s}, 60 \mathrm{~s}, 120 \mathrm{~s})$ at room temperature to find out the optimum result regarding gold surface roughness.

\subsection{PEDOT:PSS Coating Formation}

Electrodes were coated with $1 \mu \mathrm{m}$ thick PEDOT:PSS by electropolymerization for $400 \mathrm{~s}$ at $5 \mu \mathrm{A} / \mathrm{mm}^{2}$ constant current density from $10 \mathrm{mMol}$ 3,4-ethylenedioxythiophene (EDOT) and $2 \%$ weight PSS (poly(styrenesulfonate) [4]. The final flexible electrode array with PEDOT:PSS coating is shown in Figure 2.

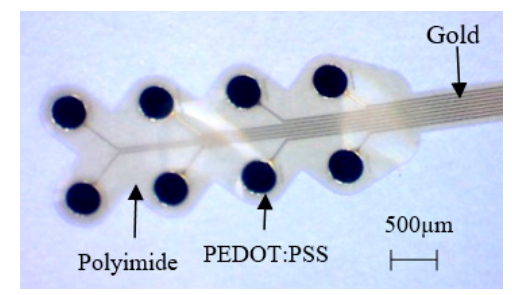

Figure 2. PEDOT:PSS coated test electrode array.

\subsection{Electrochemical Modification of PEDOT:PSS Coating}

PEDOT:PSS coating on gold microelectrode was electrochemically modified by applying cyclic voltammetry within water electrolysis window of PEDOT:PSS (+0.6 V to $-0.9 \mathrm{~V})$ [5]. PEDOT:PSS coated $\mathrm{Au}, \mathrm{Ag} / \mathrm{AgCl}, \mathrm{Pt}$ and Ringer's solution were used as working, reference and counter electrodes and electrolyte respectively. Three electrodes were immersed in electrolyte solution. Voltage of working electrode was scanned with respect to a constant potential of reference electrode while the current between counter electrode to working electrode through electrolyte solution was measured by cyclic voltammetry tool. In total 5 cyclic voltammetry scan cycles were applied for each electrode at scan rate of $100 \mathrm{mV} / \mathrm{s}$.

\section{Result}

\subsection{Stability Improvement of PEDOT:PSS Coating}

Selective iodine etching created a rough porous surface. Figure 3a,b show scanning electron microscopy (SEM) image of electrode surface without etching and after iodine etching respectively. PEDOT:PSS molecules went inside the pores and trenches and thus created a strong mechanical bond as shown in Figure 3c. 


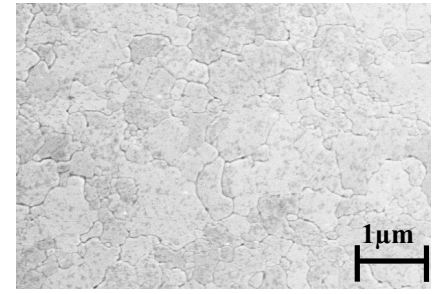

(a)

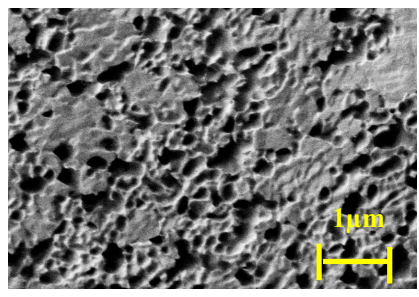

(b)

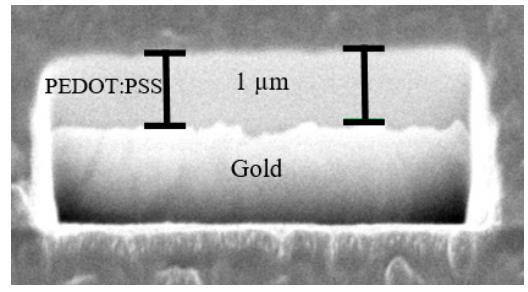

(c)

Figure 3. SEM image: (a) gold surface without etching; (b) Porous surface after iodine etching; (c) Bonding between PEDOT:PSS and iodine-etched gold surface (vertical cut through electrode).

Stability of PEDOT:PSS coating was tested by applying strong ultrasonic vibration in a water bath at $35 \mathrm{KHz}$ frequency with $300 \mathrm{~W}$ power. Series of tests were performed by gradually increasing the time from $1 \mathrm{~min}$ to $11 \mathrm{~min} .1 \mu \mathrm{m}$ thick coating on iodine etched $(0.05 \mathrm{~mol} / \mathrm{L}, 120 \mathrm{~s}$, etch rate $250 \mathrm{~nm} / \mathrm{min}$ ) electrodes showed 100\% stability compared to non-etched electrodes. No delamination of coating was noticed after $11 \mathrm{~min}$ of strong ultrasonic test. Conversely similar coating on non-etched electrode delaminated considerably after $7 \mathrm{~min}$ ultrasonic test. Figure $4 \mathrm{a}, \mathrm{b}$ show the condition of an iodine etched sample after $11 \mathrm{~min}$ and a non-etched sample after 7 min ultrasonic test respectively. The relative change of PEDOT:PSS coated surface after each step of ultrasonic test was additionally investigated by electrochemical impedance spectroscopy (EIS). Any change in the surface e.g., peels of coating increases electrode impedance. Figure 4c shows that average impedance ( $n=8$ electrodes) of non-etched electrodes increased 2.5 M $\Omega$ (very high increment) after 7 min ultrasonic test that indicated considerable delamination of coating. The small increment of impedance of iodine etched electrodes was due to mechanical modification of coating surface during strong ultrasonic test. It was not caused by delamination of coating. Therefore rough surface area of etched electrode increased adhesion of PEDOT:PSS coating.

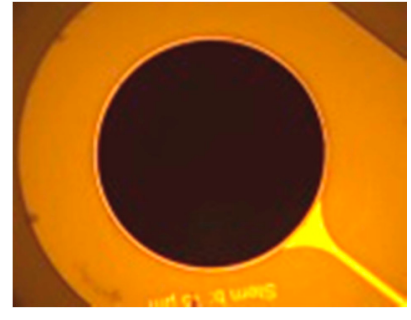

(a)



(b)

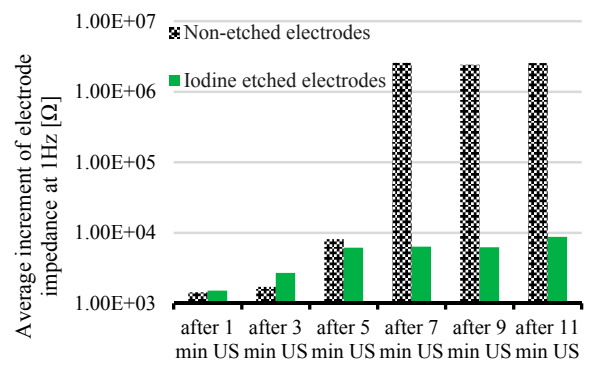

(c)

Figure 4. The condition of PEDOT:PSS coating: (a) iodine etched electrode after 11 min ultrasonic test; (b) non-etched electrode after 7 min ultrasonic test; (c) Average increment of electrode impedance during ultrasonic test of PEDOT:PSS (ultrasonic test abbreviated as US); non-etched electrode impedance increased $2.5 \mathrm{M} \Omega$ after 7 min ultrasonic test.

\subsection{Electrochemical Modification of PEDOT:PSS Surface}

Effect of electrochemical modification of PEDOT:PSS surface was examined through electrochemical impedance spectroscopy (EIS) measurement before activation and right after cyclic voltammetry activation. The samples were cleaned and dried and then EIS measurement was performed again to check the sustainability of modification process. Figure 5 a shows that electrode impedance reduces about 1.5 times and impedance phase near $1 \mathrm{KHz}$ frequency changed towards resistive behavior after cyclic voltammetry activation. The effect sustained after $1 \mathrm{~h}$ of activation. Stimulation capacity of the electrodes changed due to this surface modification. It was tested by applying biphasic charge-balance current pulse to the electrodes in Ringer's solution. Figure 5b shows that electrode polarization reduced considerably $(100 \mathrm{mV} ; 33 \%)$ during stimulation for cyclic voltammetry activated electrode compared to non-activated electrode. This indicates an increment of charge injection capacity of electrochemically modified electrodes during neural stimulation. 


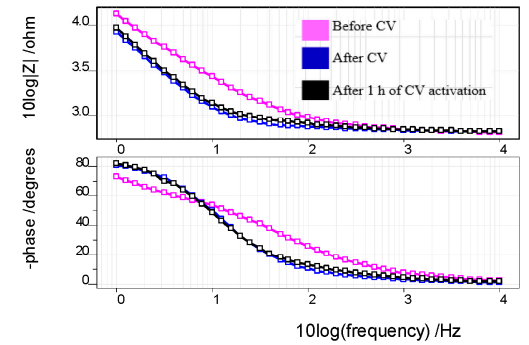

(a)

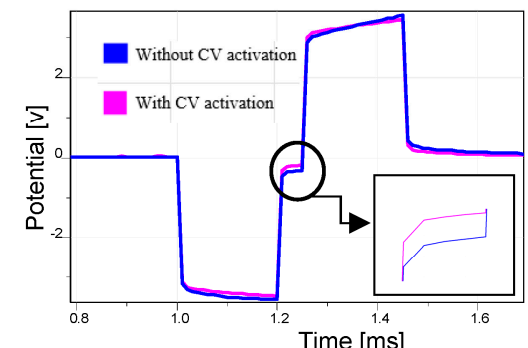

(b)

Figure 5. (a) Impedance magnitude and phase curve of PEDOT:PSS coated gold electrode before, right after $\mathrm{CV}$ activation and $1 \mathrm{~h}$ after $\mathrm{CV}$ activation; (b) electrode polarization after biphasic current pulse for $\mathrm{CV}$ activated and non-activated PEDOT:PSS coated electrode; electrode polarization reduced $100 \mathrm{mV}$ (cyclic voltammetry abbreviated as $\mathrm{CV}$ ).

\section{Conclusions}

The iodine etching process guarantees the use of PEDOT:PSS coated gold microelectrodes for long-term stable neural interfaces. Surface roughness depends on etching time and iodine solution concentration. $1 \mu \mathrm{m}$ thick PEDOT:PSS coating showed 100\% stability on gold electrodes etched in $0.05 \mathrm{~mol} / \mathrm{L}$ iodine solution for $120 \mathrm{~s}$. The cyclic voltammetry activation process significantly reduces electrode impedance and polarization during stimulation; which are the key factors for neural signal recording and high charge injection capacity during stimulation. These improvements made PEDOT:PSS as a promising electrode coating material for long-term stable bidirectional neural interfaces.

Acknowledgments: This work was supported by Priority Program SPP 1665 "Resolving and Manipulating Neuronal Networks in the Mammalian Brain-From Correlative to Causal Analysis" of the DFG (German Research Foundation) and partially by the German Excellence Initiative via the Creative Unit "I-See-The Artificial Eye: Chronic Wireless Interface to the Visual Cortex".

Conflicts of Interest: The authors declare no conflict of interest.

\section{References}

1. Venkatraman, S.; Hendricks, J.; King, Z.A.; Sereno, A.J.; Richardson-Burns, S.; Martin, D.; Carmena, J.M. In Vitro and In Vivo Evaluation of PEDOT Microelectrodes for Neural Stimulation and Recording. IEEE Trans. Neural Syst. Rehabil. Eng. 2011, 19, 307-316, doi 10.1109/TNSRE.2011.2109399.

2. Mandal, H.S.; Kastee, J.S.; McHail, D.G.; Rubinson, J.F.; Pancrazio, J.J.; Dumas, T.C. Improved Poly(3,4-Ethylenedioxythiophene) (PEDOT) for Neural Stimulation. Neuromodulation 2015, 18, 657-663, doi:10.1111/ner.12285.

3. Schander, A.; Stemmann, H.; Tolstosheeva, E.; Roese, R.; Biefeld, V.; Kempen, L.; Kreiter, A.K.; Lang, W. Design and fabrication of novel multi-channel floating neural probes for intracortical chronic recording. Sens. Actuators A Phys. 2016, 247, 125-135, doi:10.1016/j.sna.2016.05.034.

4. Schander, A.; Teßmann, T.; Strokov, S.; Stemmann, H.; Kreiter, A.K.; Lang, W. In Vitro evaluation of the long-term stability of PEDOT:PSS coated microelectrodes for chronic recording and electrical stimulation of neurons. In Proceedings of the 38th Annual International Conference of the IEEE Engineering in Medicine and Biology Society (EMBC), Orlando, FL, USA, 16-20 August 2016.

5. Cogan, S.F. Neural stimulation and recording electrodes. Annu. Rev. Biomed. Eng. 2008, 10, 275-309, doi:10.1146/annurev.bioeng.10.061807.160518.

(C) 2017 by the authors. Licensee MDPI, Basel, Switzerland. This article is an open access article distributed under the terms and conditions of the Creative Commons Attribution (CC BY) license (http://creativecommons.org/licenses/by/4.0/). 